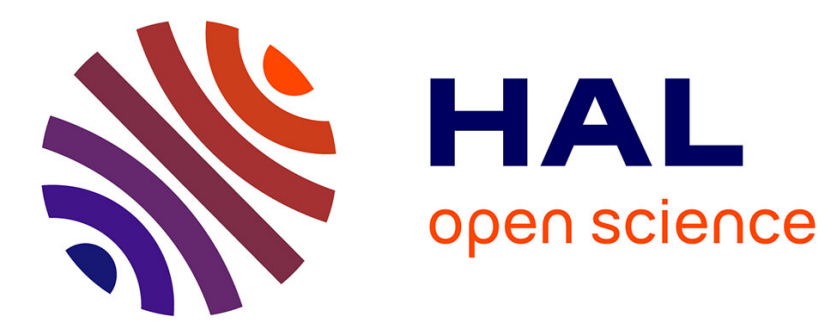

\title{
Linear study of the precessional fishbone instability
}

M Idouakass, M Faganello, Herbert L. Berk, Xavier Garbet, S Benkadda

\section{To cite this version:}

M Idouakass, M Faganello, Herbert L. Berk, Xavier Garbet, S Benkadda. Linear study of the precessional fishbone instability. 2016. hal-01360957

\section{HAL Id: hal-01360957 https://hal.science/hal-01360957}

Preprint submitted on 9 Sep 2016

HAL is a multi-disciplinary open access archive for the deposit and dissemination of scientific research documents, whether they are published or not. The documents may come from teaching and research institutions in France or abroad, or from public or private research centers.
L'archive ouverte pluridisciplinaire HAL, est destinée au dépôt et à la diffusion de documents scientifiques de niveau recherche, publiés ou non, émanant des établissements d'enseignement et de recherche français ou étrangers, des laboratoires publics ou privés. 


\section{Linear study of the precessional fishbone instability}

M. Idouakass ${ }^{1}$, M. Faganello ${ }^{1}$, H. L. Berk ${ }^{2}$, X. Garbet ${ }^{3}$, S. Benkadda ${ }^{1}$

${ }^{1}$ Aix Marseille Université, CNRS, PIIM UMR 7345, 13397 Marseille, France

${ }^{2}$ Institute for Fusion Studies, University of Texas, USA

${ }^{3}$ CEA, IRFM, 13115, St Paul lès Durance, France

(Dated: 1 August 2016)

The precessional fishbone instability is an $m=n=1$ internal kink mode destabilized by a population of trapped energetic particles. The linear phase of this instability is studied here, analytically and numerically, with a simplified model. This model uses the reduced magneto-hydrodynamics (MHD) equations for the bulk plasma and the Vlasov equation for a population of energetic particles with a radially decreasing density. A threshold condition for the instability is found, as well as a linear growth rate and frequency. It is shown that the mode frequency is given by the precession frequency of the deeply trapped energetic particles at the position of strongest radial gradient. The growth rate is shown to scale with the energetic particle density and particles energy while it is decreased by continuum damping.

PACS numbers: 52.35.Py, 52.35.-g, 52.25.Dg, 52.35.Qz

\section{INTRODUCTION}

The resonant interaction between a distribution of particles and waves evolving in a plasma is one of the peculiarities of the kinetic dynamics of plasmas. It can excite or damp waves, depending on the sign of the gradient of the distribution of particles in momentum direction. Indeed, when in the neighborhood of a resonance, the distribution of the faster particles is less than that of slower particles, there is a net transfer of energy from the wave to the plasma, leading to the well-known phenomenon of Landau damping ${ }^{1}$. In contrast, it is possible to excite a wave by taking energy from the resonating particles, in the case where there is a reversed gradient in the distribution function, such as when a beam of energetic particles is present in a cold plasma. This case is known as the bump-on-tail instability (referring to a bump of energetic particles on the tail of the distribution function of the thermal particles). This instability has been studied excluding $^{2}$ or including ${ }^{3}$ in the model a finite dissipation for the wave. It was shown that without dissipation, the nonlinear dynamics would flatten the gradient around the position of the wave-particle resonance, thus leading to a saturation of the mode ${ }^{2,3}$. It was later shown that, with dissipative processes, that saturation would also occur, but there was the possibility of a change in frequency of the mode (called chirping) after its saturation in amplitude. This chirping was seen in particle simulations to be associated with the dynamics of energetic particles and the formation of coherent structures in the phasespace, moving in the momentum direction ${ }^{3-5}$. Such dynamics in phase space have been studied, not only for the bump-on-tail case, but also for various Energetic Particle Modes (EPMs), be it for specific modes such as the Alfvén waves ${ }^{6}$ or in a more general way ${ }^{7}$.

In the context of Tokamak Physics, where an ultimate goal is to obtain a burning plasma, energetic particles play a key role in heating the thermal plasma. The way they deposit their energy onto the plasma is thus critical.
These energetic particles can be fusion produced alpha particles as well as energetic particles resulting from various heating mechanisms. An important concern for this energy deposition is that there are resonant instabilities that tend to take energy from energetic particles and, at the same time, to expel these particles from the plasma core, before they can transfer their energy to the plasma. One of these instabilities is the fishbone instability.

Fishbones were first observed in the Poloidal Divertor Experiment (PDX) tokamak ${ }^{8}$ in 1983. This instability was observed to have a kink-like mode structure, with dominant mode numbers $m=n=1$, and to occur in repetitive bursts, where the mode frequency decreases by a factor of about 2 during each burst. It was seen when the Neutral Beam Injection (NBI) was active, in a direction nearly perpendicular to the magnetic field. The mode frequency was comparable to the precession frequency of the deeply trapped particles generated by the NBI. Furthermore, these bursts were associated with losses of fast particles, i.e. an outward radial displacement of these particles.

Two different theoretical interpretations were given for two different regimes. The first regime ${ }^{9}$, of precessional fishbones, refers to the case where the mode frequency $\omega$ is much greater than the diamagnetic frequency $\omega_{*, i}$ associated with the radial pressure gradient of the thermal ions. In that regime, the trapped energetic particles destabilize a $m=n=1$ mode with the frequency of their precessional motion $\omega_{D}$. This mode is purely kinetic and exists only when energetic particles are present, otherwise it is strongly damped by the Alvfén continuum ${ }^{10,11}$. The second regime ${ }^{12}$ refers to a mode with frequency $\omega \approx \omega_{*, i} \approx \omega_{D}$. In that regime, the energetic particles excite a mode that already exists, a marginally stable internal kink mode that oscillates at the ion diamagnetic frequency.

It should be stressed that, in a torus, an $m=n=1$ mode with an electric potential proportional to the minor radius within the $q=1$ surface (kink-like mode) corresponds to a rigid displacement of the core region, without 
a bending of the field lines. Thus the mode, minimizing the work that should be done against the magnetic tension, is the easiest one to excite. Here $q$ is, as usual, the safety factor.

One can make a few simplifications to analyze the essence of the precessional branch of the instability. As it was observed in the work by McGuire ${ }^{8}$, the mode frequency is close to the precessional frequency of deeply trapped energetic ions. It is thus possible to simplify the problem by assuming that all the particles are deeply trapped. With this simplification, it is possible to reduce the particle dynamics to a two-dimensional phase space where the particle-wave interaction occurs only through the electrostatic part of the wave field.

Again, with this simplification, and for a sufficiently large magnetic field, the toroidal angular momentum $P_{\varphi}$, which is a constant of motion in the unperturbed field, is approximately the poloidal magnetic flux (to within a constant factor) and hence, the minor radius $r$ is an approximate constant of motion. This is an important feature, as then a decrease in fast particle density along radius can be seen as a positive gradient of the distribution function in the toroidal momentum direction ${ }^{13}$. A decreasing density of deeply trapped energetic particles play exacly the same role of a bump on the tail, in the context of electrostatic waves. Many different works have studied this problem, retaining more effects than we shall use. The more general effects can include: gyrokinetic ions $^{14}$, vertical plasma elongation ${ }^{15}$, or a different kind of energetic particles, such as barely trapped/circulating electrons $^{16,17}$.

In this paper, we will study the linear phase of the precessional fishbone instability in the simplified model discussed above. This model, due to its simplicity and reduced dimensionality, has the great advantage of being analytically integrable in linear theory. It can thus give direct indication of the roles played by the different mechanisms that drive or suppress the instability. Furthermore it can serve as a benchmark for nonlinear codes, at least when the hypotheses are fulfilled. In section II, we will introduce the model for the bulk plasma as well as for the fast particles. This model is in the spirit of the one developed by Ödblom et al. ${ }^{10}$, with the notable difference that we have here a continuous radial distribution of fast particles but a single value for the magnetic moment, where they use a Heavyside function for the radial distribution and a Maxwellian for the perpendicular velocity distribution. In section III, the linear analysis of the model is presented, and an approximate analytic value is obtained for the instability threshold, the linear growth rate and the frequency. Section IV describes the numerical implementation of the model, and section $\mathrm{V}$ discusses the linear numerical benchmarking of the code. The summary and conclusions are found in section VI.

\section{MODEL}

The model will treat differently the dynamics of the thermal plasma and the dynamics of the energetic particles. In this model, we consider a small inverse aspect ratio tokamak where $\varepsilon=r / R \ll 1$ ( $R$ is the major radius and $r$ the minor radius). The thermal plasma will be described by the reduced MHD equations in a cylindrical geometry, meaning that in accord with the small inverse aspect ratio approximation, the toroidal curvature effects can be neglected, exept for the interaction with energetic particles. The fast particles will be described kinetically and the toroidal curvature, which is crucial for the dynamics of the energetic particles, will be included. The curvature will be used in the description of the phase space evolution and, together with the energetic particle pressure, in the vorticity equation.

\section{A. MHD description of the thermal plasma}

Let us define the magnetic field and the fluid velocity field as

$$
\begin{gathered}
\mathbf{B}=\mathbf{B}_{T}+\nabla \psi \times \mathbf{e}_{z} \\
\mathbf{v}=\nabla \phi \times \mathbf{e}_{z}=\frac{1}{B_{T}} \nabla \phi_{e l} \times \mathbf{e}_{z}
\end{gathered}
$$

where $\phi_{e l}$ is the electric potential, $\phi$ the stream function, $\mathbf{B}_{T}$ is the axial guide field in cylindrical geometry $\mathbf{B}_{T}=B_{T} \mathbf{e}_{z}$ and $\psi=A_{\|}$where $A_{\|}$is the axial component of the vector potential. The plasma density variations $\left(\rho(\boldsymbol{r})=\rho_{0}\right)$ and the thermal pressure effects are neglected.

Keeping in the effects of energetic particle pressure, and noting that, as only deeply trapped particles are considered, the perpendicular component of the pressure is much larger than the parallel one $P_{\perp, h} \gg P_{\|, h}$, the following two equations of the ideal reduced MHD are obtained

$$
\begin{gathered}
\frac{\partial \psi}{\partial t}=(\mathbf{B} \cdot \nabla) \phi \\
\frac{\partial}{\partial t} \Omega+(\mathbf{v} \cdot \nabla) \Omega=\frac{1}{\mu_{0} \rho_{0}}(\mathbf{B} \cdot \nabla) \Delta_{\perp} \psi \\
-(\mathbf{b} \times \boldsymbol{\kappa}) \cdot \nabla \frac{P_{\perp, h}}{B_{T}}
\end{gathered}
$$

where $\boldsymbol{b}$ is the unit vector along the equilibrium magnetic field, $\boldsymbol{\kappa}$ mimics the toroidal curvature, $\Omega$ is the vorticity $\Omega=\Delta_{\perp} \phi$ and $\Delta_{\perp}$ is the cylindrical laplacian in the direction perpendicular to the magnetic guide field along $e_{z}$. 
The fields are expanded in an equilibrium part and a perturbation as $\psi=\psi_{e q}+\hat{\psi}, \phi=\phi_{e q}+\hat{\phi}$. The perturbations are expressed in terms of the following Fourier series

$$
(\hat{\phi}, \hat{\psi})=\sum_{m, n}\left(\hat{\phi}_{m, n}(r), \hat{\psi}_{m, n}(r)\right) e^{-i \omega t+i n \varphi-i m \theta}+c . c .
$$

where $\theta$ is the poloidal angle and $\varphi=\frac{2 \pi z}{L_{z}}=\frac{z}{R}$ is equivalent to the toroidal angle, implying a periodic axial direction $z$. For the linear study considered here, only the mode numbers $m=n=1$ are relevant. As such, the perturbed fields become

$$
(\hat{\phi}, \hat{\psi})=\left(\hat{\phi}_{1,1}(r), \hat{\psi}_{1,1}(r)\right) e^{-i \omega t+i \varphi-i \theta}+c . c .
$$

In the following, the "hat" notation, the indices, and the explicit radial dependence are dropped for simplicity.

Equations (3) and (4) are now linearized assuming that there is no equilibrium flow $\left(\phi_{e q}=0\right)$

$$
\begin{gathered}
-i \omega \psi=i \frac{B_{T}}{R_{0}}\left(1-\frac{1}{q}\right) \phi \\
-i \omega \Omega=i \frac{B_{T}}{\mu_{0} \rho_{0} R_{0}}\left(1-\frac{1}{q}\right) \Delta_{\perp} \psi \\
+\frac{B_{T}}{\mu_{0} \rho_{0} R_{0}}\left(1-\frac{1}{q}\right) \frac{\phi}{r} \partial_{r} J_{e q}-(\boldsymbol{b} \times \boldsymbol{\kappa}) \cdot \nabla \frac{P_{\perp, h}}{B_{T}}
\end{gathered}
$$

where $J_{e q}$ is the equilibrium toroidal current, which can be expressed as $J_{e q}=\Delta_{\perp} \psi_{e q}=\frac{1}{r} \partial_{r} r \partial_{r} \psi_{e q}$.

Combining equations (7) and (8) yields

$\frac{1}{r^{2}} \partial_{r}\left(r^{3}\left[\omega_{A}^{2}(r)-\omega^{2}\right] \partial_{r}\left(\frac{\phi}{r}\right)\right)=-i \omega \frac{B_{T}}{\rho_{0}}(\boldsymbol{b} \times \boldsymbol{\kappa}) \cdot \nabla \frac{P_{\perp, h}}{B_{T}}$

which, once integrated with respect to radius, gives

$$
\left[\omega_{A}^{2}(r)-\omega^{2}\right] \partial_{r}\left(\frac{\phi}{r}\right)=-i \omega \tilde{\rho}
$$

where $\tilde{\rho}=\frac{1}{r^{3}} \int_{0}^{r} r^{2} \frac{B_{T}}{\rho_{0}}(\boldsymbol{b} \times \boldsymbol{\kappa}) \cdot \nabla \frac{P_{\perp, h}}{B_{T}} d r$ is the fast particle contribution term and $\omega_{A}(r)=\frac{v_{A, T}}{R}\left(1-\frac{1}{q}\right)$ is the Alfvén frequency associated with the mode helicity.

\section{B. Fast particle response}

We now describe the kinetic evolution of the fast particles, which satisfies the Vlasov equation

$$
\partial_{t} f-\{H, f\}=0
$$

where $\{g, h\}=\partial_{\varphi} g \partial_{P_{\varphi}} h-\partial_{\varphi} h \partial_{P_{\varphi}} g$ are the Poisson brackets, which operate on the toroidal angle $\varphi$ and its conjugate momentum variable $P_{\varphi}$. The fast particle distribution $f=F_{e q}+\delta f$ is decomposed into an equilibrium part $F_{e q}$ and a perturbation $\delta f$. The Hamiltonian
$H=H_{e q}+H_{1}$ is also decomposed into an equilibrium part $H_{e q}$ and a perturbation $H_{1}$.

Then, it should be noted that we are considering a population of deeply trapped fast particles, for which $v_{\|}$ is negligible compared with $v_{\perp}$ and $\frac{e}{M} \psi_{e q}$, where parallel and perpendicular correspond to directions relative to the equilibrium magnetic field, $e$ is the particle charge and $M$ its mass.

These considerations give a few important results. The first one is that all fast particles lie close to the $\theta=0$ plane (the horizontal mid-plane of the tokamak, outside of the magnetic axis). Therefore, all interactions of the energetic particles with modes described as $g(r, \theta, \varphi)=\Sigma_{m, n=0}^{\infty} g_{m, n}(r) e^{-i \omega t+i n \varphi-i m \theta}+$ cc can be taken as $g(r, \theta=0, \varphi)=\Sigma_{m, n=0}^{\infty} g_{m, n}(r) e^{-i \omega t+i n \varphi}+\mathrm{cc}$. Furthermore the perturbed Hamiltonian simplifies, from the form $H_{1}=e\left(\phi-v_{\|} \delta A_{\|}\right)$to $H_{1}=e \phi$, where $\delta A_{\|}=$ $A_{\|}-A_{\|, e q}$. Then the Hamiltonian is of the form

$$
H=H_{e q}\left(P_{\varphi}, \mu\right)+e \phi_{e l, n}\left(P_{\varphi}\right) e^{-i \omega t+i n \varphi}
$$

Here $P_{\varphi}$ is the toroidal momentum, and it is equivalent to the radial variable. As it is defined $P_{\varphi}=e R \psi$, we find

$$
\frac{\partial P_{\varphi}}{\partial r}=-\frac{e r B_{T}}{q}
$$

The equilibrium Hamiltonian is simply given by $H_{e q}=$ $\mu B$ where $\mu$ is the magnetic moment.Thus the equilibrium precessional frequency of particles is simply

$$
\omega_{D}=\frac{\partial H_{e q}}{\partial P_{\varphi}}=\frac{\mu B_{T} q}{\omega_{C} M R r}
$$

where $\omega_{C}$ is the cyclotron frequency of the particles considered. It can be seen here that, for a relatively flat $q$-profile, the precession frequency will decrease with radius.

\section{LINEAR THEORY}

\section{A. Linear particle response}

Using this Hamiltonian in Eq. (11), one gets the linearized Vlasov equation

$$
\partial_{t} \delta f=\omega_{D} \partial_{\varphi} \delta f-\partial_{\varphi} e \phi_{e l} \partial_{P_{\varphi}} F_{e q}
$$

which, once solved, gives for the perturbation

$$
\delta f=\frac{e \phi_{e l} \partial_{P_{\varphi}} F_{e q}}{\omega_{D}-\omega}
$$

Now, the shape of the MHD mode must be taken into consideration. The dominant mode is kink-like, which means that the mode numbers are $m=n=1$ and, well inside the $q=1$ surface, the electric potential is, at the lowest order, proportional to the radius

$$
\phi_{e l}(r, \theta, \varphi)=r\left(\frac{\phi_{e l}}{r}\right)_{r=r_{b}} e^{-i \omega t+i \varphi-i \theta}
$$


Here $r_{b}$ is an arbitrary radius that is small enough compared to $r_{*}$, the radial position of the $q=1$ surface, so that $\frac{\phi_{e l}}{r}$ has already reached the asymptotic value it has inside the core. Furthermore if we suppose that the energetic particles remain inside the volume defined by $r_{b}$, the radial shape of the electric potential given by Eq.17, can be used for determining $\delta f$ via Eq.16.

The contribution of fast particles to the MHD dynamics is given by

$$
\tilde{\rho}=\frac{B_{T}}{r^{3} \rho_{0}} \int_{0}^{r} r^{2} d r(\boldsymbol{b} \times \boldsymbol{\kappa}) \cdot \nabla \frac{P_{\perp, h}}{B_{T}}
$$

where

$$
(\boldsymbol{b} \times \boldsymbol{\kappa}) \cdot \nabla \approx e \frac{\omega_{D}}{\mu} \partial_{\varphi}
$$

which, it should be pointed out, is independent of $\mu$. The perturbed pressure is given by

$$
\begin{array}{r}
P_{\perp, h}=\int f \mu B_{T} d^{3} v=\int\left(F_{e q}\left(\mu, v_{\|}, P_{\varphi}\right)\right. \\
\left.+\delta f\left(\mu, v_{\|}, P_{\varphi}, \varphi\right)\right) \mu B_{T} d^{3} v
\end{array}
$$

where $d^{3} v=2 \pi v_{\perp} d v_{\perp} d v_{\|}=2 \pi \frac{B_{T}}{M} d \mu d v_{\|}$so that

$$
\begin{gathered}
\tilde{\rho}=\frac{2 \pi e B_{T}^{2}}{M r^{3} \rho_{0}} \int_{0}^{r} r^{2} d r \frac{\omega_{D}}{\mu} \partial_{\varphi} \int \mu f d \mu d v_{\|} \\
=\frac{2 \pi i e B_{T}^{2}}{M r^{3} \rho_{0}} \int_{0}^{r} r^{2} d r \frac{\omega_{D}}{\mu} \int \mu \delta f d \mu d v_{\|} \\
\tilde{\rho}=\frac{2 \pi i e B_{T}^{2}}{M r^{3} \rho_{0}} \int_{0}^{r} r^{2} d r \frac{\omega_{D}}{\mu} \times \\
\int \mu \frac{e r\left(\frac{\phi_{e l}}{r}\right)_{r=r_{b}} \partial_{r} F_{e q}}{\omega_{D}-\omega} \frac{\partial r}{\partial P_{\varphi}} d \mu d v_{\|}
\end{gathered}
$$

Now let us define a general form for the equilibrium distribution function

$$
F_{e q}=A \delta\left(\frac{\mu}{\mu_{*}}-1\right) \delta\left(\frac{v_{\|}}{v_{\perp}}\right) F_{r}(r)
$$

where $\delta$ is the Dirac delta function and we have chosen the single value of magnetic moment to be $\mu_{*}$. In this way Eq.22 reads

$\tilde{\rho}=-\frac{2 \sqrt{2} \pi i e B_{T}^{3 / 2} A\left(\frac{\phi_{e l}}{r}\right)_{r=r_{b}} \mu_{*}^{3 / 2}}{M^{3 / 2} r^{3} \rho_{0}} \int_{0}^{r} q r^{2} d r \frac{\omega_{D *} \partial_{r} F_{r}(r)}{\omega_{D *}-\omega}$

Changing the integration variable to $y=\frac{r}{r_{*}}$ where $r_{*}$ is the radius of the $q=1$ surface, and expressing $\omega_{D *}$ as $\omega_{D *}=\frac{q r_{*}}{r T}$ where $T$ is a characteristic period $T=$ $\frac{1}{\omega_{D *}\left(r_{*}\right)}$, one gets

$\tilde{\rho}=-\frac{2 \sqrt{2} \pi i e A\left(\frac{\phi}{r}\right)_{r=r_{b}} \mu_{*}^{3 / 2} B_{T}^{5 / 2}}{M^{3 / 2} y^{3} r_{*} \rho_{0}} \int_{0}^{y} \frac{y^{\prime 2} q^{2} \partial_{y} F_{y^{\prime}}\left(y^{\prime}\right)}{q-\omega T y^{\prime}} d y^{\prime}$

\section{B. MHD response and dispersion relation}

Starting back from Eq. (10), a streched variable can be defined as $x=\left(r-r_{*}\right) / \epsilon$ around the position where all variations of $\phi / r$ are located, i.e. around the $q=1$ surface. These localized variations allow us to take the value of $r$ in Eq. (25) to be $r=r_{*}$ i.e. $y=1$. As a result, one can integrate in radius around the $q=1$ surface, so that

$$
\left[\frac{\phi}{r}\right]_{r=r_{b}}^{r=r_{b}+2 \Delta_{r}}=-\left(\frac{\phi}{r}\right)_{r=r_{b}}=\epsilon \int_{-\Delta_{r} / \epsilon}^{\Delta_{r} / \epsilon} \frac{-i \omega \tilde{\rho}\left(r_{*}\right)}{\omega_{A}^{2}-\omega^{2}} d x
$$

Here $\Delta_{r}$ is then defined as $\Delta_{r}=r_{*}-r_{b}$ and $\phi$ goes to zero at $r=r_{b}+2 \Delta_{r}$.

Linearizing $\omega_{A}$ so that $\omega_{A}=\epsilon \omega_{A}^{\prime} x$ one finds

$$
-\left(\frac{\phi}{r}\right)_{r=r_{b}}=\frac{\pi}{\omega_{A}^{\prime}} \tilde{\rho}\left(r_{*}\right)
$$

which translates to the following dispersion relation

$$
i=-K \int_{0}^{1} \frac{y^{2} q^{2} \partial_{y} F_{y}(y)}{q-\omega T y} d y
$$

where

$$
K=\frac{2 \sqrt{2} \pi^{2} \mu_{*}^{3 / 2} B_{T}^{5 / 2} e A}{M^{3 / 2} r_{*} \rho_{0} \omega_{A}^{\prime}}
$$

is a factor taking into account the energetic content of the fast particles as well as the thermal plasma, geometric and magnetic parameters.

\section{Threshold, frequency and growth rate}

In order to perform an analytic treatment, some additionnal assumptions need to be made about the distribution function. As it was mentioned, all fast particles are assumed to be well contained within the $q=1$ surface, and in addition it is assumed that the distribution function's radial gradient goes to zero at the magnetic axis. Furthermore, in order to be able to get an analytic solution, it is assumed that $q(y) \simeq 1$ in the entire integration domain.

\section{General distribution}

Taking a $F_{y}$ in a form such that

- it is monotonic

- its gradient is centered around $y=y_{0}$

- its gradient is peaked enough around $y=y_{0}$ 
one can solve approximately the dispersion relation, getting a threshold condition

$$
K_{0}=-\frac{1}{\left.\pi y_{0}^{3} F_{y}^{\prime}\right|_{y_{0}}} \quad ; \quad \omega_{0}=\omega_{D *}\left(y_{0}\right)
$$

This solution shows that the threshold value $K_{0}$ is inversely proportional to the maximum (negative) value of the distribution function's gradient, meaning that the mode will be more easily unstable if the gradient of the distribution function is more peaked. Furthermore, as expected, the mode frequency is consistent with the precessional frequency of particles at the location of the maximum gradient value.

\section{Specific distribution}

Let us now give an analytic profile for the radial distribution

$$
F_{y}(y)=\frac{1}{2}\left(1-\operatorname{erf}\left(\beta\left(y-y_{0}\right)\right)\right.
$$

with a $\beta$ and a $y_{0}$ chosen so that $F_{y}$ goes to a constant at $y=0$ and $y=1$ (giving the "narrow gradient condition" $\left.\beta y_{0} \gg 1 ;\left(1-y_{0}\right) \beta \gg 1\right)$. This distribution has a radial gradient

$$
\partial_{y} F_{y}=-\frac{\beta}{\sqrt{\pi}} e^{-\beta^{2}\left(y-y_{0}\right)^{2}}
$$

which allows for an exact solution of the dispersion relation eq. (28) to be expressed as

$$
\begin{array}{r}
i=\frac{K}{\omega T} \int_{0}^{1} y F_{y}^{\prime}(y) d y+\frac{K}{(\omega T)^{2}} \int_{0}^{1} F_{y}^{\prime}(y) d y \\
-\frac{K}{(\omega T)^{2}} \int_{0}^{1} \frac{F_{y}^{\prime}(y)}{1-\omega T y} d y
\end{array}
$$

which can be simplified, noticing that $\left[y F_{y}\right]_{0}^{1}=0$ and that $\int_{0}^{1} F_{y}(y) d y \simeq y_{0}$, to

$$
i=-\frac{K}{\omega T} y_{0}-\frac{K}{(\omega T)^{2}} F_{y}(0)-\frac{K}{(\omega T)^{2}} \int_{0}^{1} \frac{F_{y}^{\prime}(y) d y}{1-\omega T y}
$$

The last integral term can be redefined as

$$
\begin{aligned}
\int_{0}^{1} \frac{F_{y}^{\prime}(y) d y}{1-\omega T y} & =-\frac{\beta}{\sqrt{\pi}} \int_{0}^{1} \frac{e^{-\beta^{2}\left(y-y_{0}\right)^{2}} d y}{1-\omega T y} \\
& =-\frac{\beta}{\omega T \sqrt{\pi}} \int_{-\beta y_{0}}^{\beta\left(1-y_{0}\right)} \frac{e^{-z^{2}} d z}{X-z}
\end{aligned}
$$

where $z=\beta\left(y-y_{0}\right)$ and $X=\frac{\beta}{\omega T}\left(1-\omega T y_{0}\right)$. Using again the narrow gradient condition, the integration domain can be stretched to infinity. Here the causal prescription is chosen, meaning that $\Im(\omega)$ is taken positive (positive growth rate), giving a negative $\Im(X)$. In this case, the integral reads ${ }^{18}$

$$
\int_{-\infty}^{+\infty} \frac{e^{-z^{2}} d z}{X-z}=i \pi\left(2 e^{-X^{2}}-w(X)\right)
$$

where $w(X)$ is the Faddeeva function. The exponential term is due to the pole coming from the causal prescription. The dispersion relation is then given as :

$$
i=-\frac{K y_{0}}{\omega T}-\frac{K}{(\omega T)^{2}}+\frac{i K \beta \sqrt{\pi}}{(\omega T)^{3}}\left(2 e^{-X^{2}}-w(X)\right)
$$

We can show that the frequency at the threshold is given by $\frac{1}{y_{0} T}=\omega_{D *}\left(y_{0}\right)$ plus a small correction. Let us define $\delta=1-\omega_{0} T y_{0} \ll 1$, so that $X=\frac{\beta}{\omega_{0} T} \delta$, and expand the dispersion relation while only keeping the first order terms in $X$

$i=-\frac{K_{0} y_{0}^{2}}{1-\delta}-\frac{K_{0} y_{0}^{2}}{(1-\delta)^{2}}+\frac{i K_{0} \beta y_{0}^{3} \sqrt{\pi}}{(1-\delta)^{3}}\left(1-\frac{2 i X}{\sqrt{\pi}}\right)+O\left(X^{2}\right)$

To the lowest order, this leads to

$$
i=-2 K_{0} y_{0}^{2}\left(1-\beta^{2} y_{0}^{2} \delta\right)+i K_{0} \beta y_{0}^{3} \sqrt{\pi}
$$

from where we get

$$
K_{0}=\frac{1}{\sqrt{\pi} \beta y_{0}^{3}} \quad ; \quad \omega_{0}=\frac{1}{y_{0} T}(1-\delta) ; \quad \delta=\frac{1}{\beta^{2} y_{0}^{2}}
$$

These relations are the threshold values given for the fishbone mode in our model. Note that the correction to the frequency at threshold $\delta$ is indeed small. We can see that these relations are the same as the ones for a general distribution in Eq. 30, with a correction of order $O(\delta)$ related to the finite width of the gradient.

It is now possible to find values for the growth rate and the real frequency close to the threshold values. Let us expand the dispersion relation around the threshold values and write $K$ and $\omega$ as $K=K_{0}+\delta K$ and $\omega=$ $\omega_{0}+\delta \omega$. The dispersion relation Eq. (37), written as $i=D(K, \omega)$ reads

$i=D\left(K_{0}, \omega_{0}\right)+\left.\delta K \frac{\partial D}{\partial K}\right|_{K_{0}, \omega_{0}}+\left.\delta \omega \frac{\partial D}{\partial \omega}\right|_{K_{0}, \omega_{0}}+O\left(\delta K^{2}, \delta \omega^{2}\right)$

It is straightforward to show that $\frac{\partial D}{\partial K}=\frac{D}{K}=\frac{i}{K}$. At the same time, to zeroth order in $\delta$,

$$
\frac{\partial D}{\partial \omega}=-i \sqrt{\pi} K_{0} T \beta y_{0}^{4}-2 K_{0} T \beta^{2} y_{0}^{5}
$$

which leads to the relation

$$
i \frac{\delta K}{K_{0}}-\left(i \sqrt{\pi} K_{0} T \beta y_{0}^{4}+2 K_{0} T \beta^{2} y_{0}^{5}\right) \delta \omega=0
$$

Taking $\delta \omega=\delta \omega_{R}+i \gamma$ and separating the dispersion relation into its imaginary part and its real part we obtain

$\gamma \simeq \frac{\delta K}{K_{0}} \frac{\sqrt{\pi}}{2 \beta y_{0}} \omega_{0}+O\left(\delta K^{2}\right) \quad ; \quad \delta \omega_{R} \simeq \frac{1}{2} \frac{\sqrt{\pi}}{\beta y_{0}} \gamma+O\left(\delta K^{2}\right)$

that represent the growth rate and the real frequency correction close to the threshold. 


\section{NUMERICAL IMPLEMENTATION OF THE MODEL}

The numerical model is based on the same assumptions as the linear theory. In this case a domain decomposition is done, separating the area of interest in two regions. The first region is the core region, centered on the magnetic axis, and extending up to $r_{b}$, a radius close to $r_{*}$, that of the $q=1$ surface. It is considered that all fast particles are contained in this region. The second domain is an annular region centered around the $q=1$ surface, with $r_{b}<r<r_{b}+2 \Delta_{r}$, in which there are no fast particles. Here, a 2 dimensional code will be used instead of simply integrating numerically the linear equations, as it will serve for future nonlinear studies.

\section{A. Core region}

This region is the part of the $\theta=0$ plane that is located between the magnetic axis and the $q=1$ surface. As it was said previously, the radius is parametrized by the toroidal momentum, and therefore this region can be described kinetically in angle-action variables, the action being the toroidal momentum $P_{\varphi}$, and the angle the toroidal angle $\varphi$. As before the Hamiltonian is given by an equilibrium part $H_{0}=\mu_{*} B_{T}$ and a time varying part, i.e. the electric potential of the mode:

$$
H_{1}\left(\varphi, P_{\varphi}\right)=\operatorname{er}\left(P_{\varphi}\right)\left(\frac{\phi_{e l}(t)}{r\left(P_{\varphi}\right)}\right)_{r\left(P_{\varphi}\right)=r_{b}} e^{i \varphi}
$$

The evolution of the distribution function $f$ is given by Eq.11.

The Vlasov equation in this region is treated numerically using a semi-lagrangian scheme with the code VLAP, using a CIP-CSL method ${ }^{19,20}$. It can be seen that this is a nonlinear equation, but for the study of interest here, we will look only at the linear phase of the evolution.

For consistency with the analytic prediction, the $q$ profile will be taken as $q(r)=q_{0} \simeq 1$ inside the core region. This allows us to get an explicit relation between $P_{\varphi}$ and $r$ :

$$
P_{\varphi}(r)-P_{\varphi, 0}=-e\left(r^{2}-r_{0}^{2}\right) \frac{B_{T}}{2}
$$

where $r_{0}$ is the minimal radius of the core region, and $P_{\varphi, 0}$ is the minimal value for the toroidal momentum.

\section{B. Annular region}

This region is a narrow layer centered around $r_{*}$. By considering a single helicity response $(m=n=1)$, and the fact that its width is small compared with its length $\left(\Delta_{r} / r_{*} \ll 1\right)$, it is possible to simplify the geometry to a two dimensional slab. As there are no fast particles in this region, the dynamics is described by the usual reduced MHD model, without considering the fast particle contribution

$$
\begin{gathered}
\frac{\partial \psi}{\partial t}=[\psi, \phi]+\eta \Delta_{\perp} \psi \\
\frac{\partial}{\partial t} \Omega=[\Omega, \phi]+\left[\psi, \Delta_{\perp} \psi\right]+\nu \Delta_{\perp} \Omega
\end{gathered}
$$

where $[f, g]=\frac{1}{r_{*}} \partial_{r} f \partial_{\theta} g-\frac{1}{r_{*}} \partial_{\theta} f \partial_{r} g$. A small but finite resistivity $\eta$ and viscosity $\nu$ have been added for numerical stability.

These equations have been cast in a dimensionless form by normalizing all length scales to the $q=1$ radius $r_{*}$ (so that $r=1$ corresponds to the $q=1$ surface), all time scales to the Alfvén time, and the magnetic fields to the equilibrium magnetic field. Only the linearized equations for the mode $m=1$ are retained in our simulations. Once linearized, they read

$$
\begin{gathered}
\frac{\partial \psi}{\partial t}=i \phi \partial_{r} \psi_{e q}+\eta \Delta_{\perp} \psi \\
\frac{\partial}{\partial t} \Omega=\partial_{r} \psi_{e q} \Delta_{\perp} \psi-i \psi \partial_{r r} \psi_{e q}+\nu \Delta_{\perp} \Omega
\end{gathered}
$$

where $\frac{1}{r_{*}} \partial_{\theta}=i k_{\theta}=i$. The MHD equations in this region are treated numerically using a semi-spectral finite differences scheme with the code $\mathrm{AMON}^{21}$. It should be noted here that, in accordance with the analytic theory, the equilibrium poloidal magnetic field profile is taken linear. It is of the form $\partial_{r} \psi_{e q}=B_{\theta, e q}=\frac{B_{T} r_{*}}{R} \frac{r-r_{*}}{L_{S}}$, where $L_{S}$ is a characteristic shear length of the magnetic field.

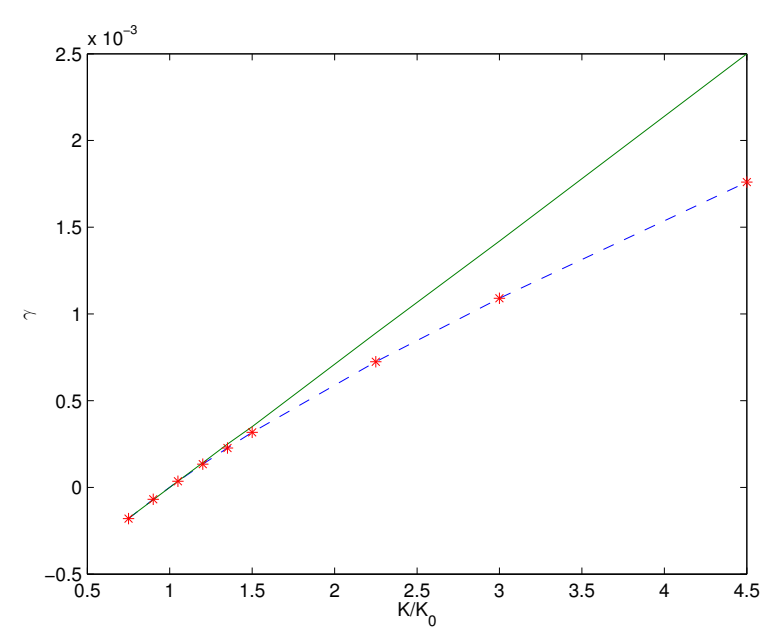

FIG. 1. Growth rate of the mode as a function of $K / K_{0}$. Solid line is the theoretical analytic result, stars linked with the dashed line are the numerical points. 


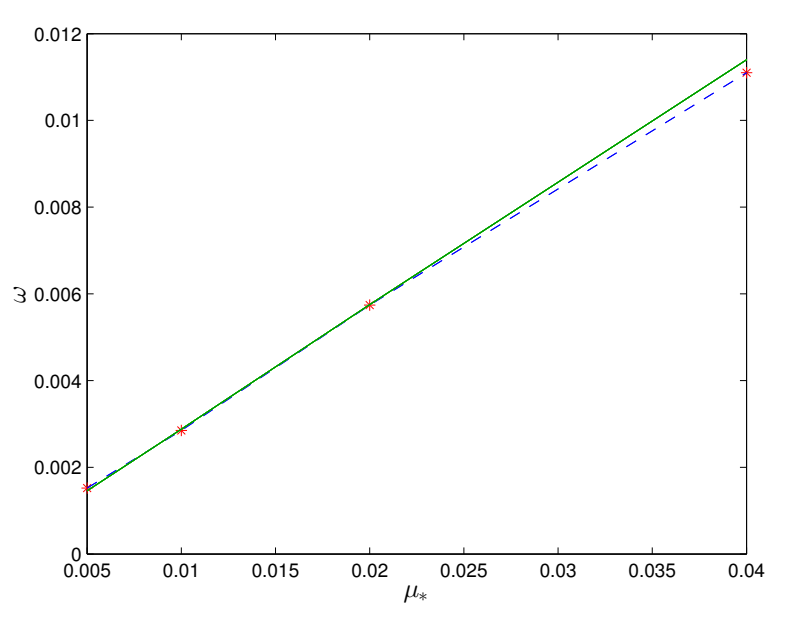

FIG. 2. Frequency of the mode as a function of $\mu_{*}$. Solid line is the theoretical result, stars linked with the dashed line are the numerical points.

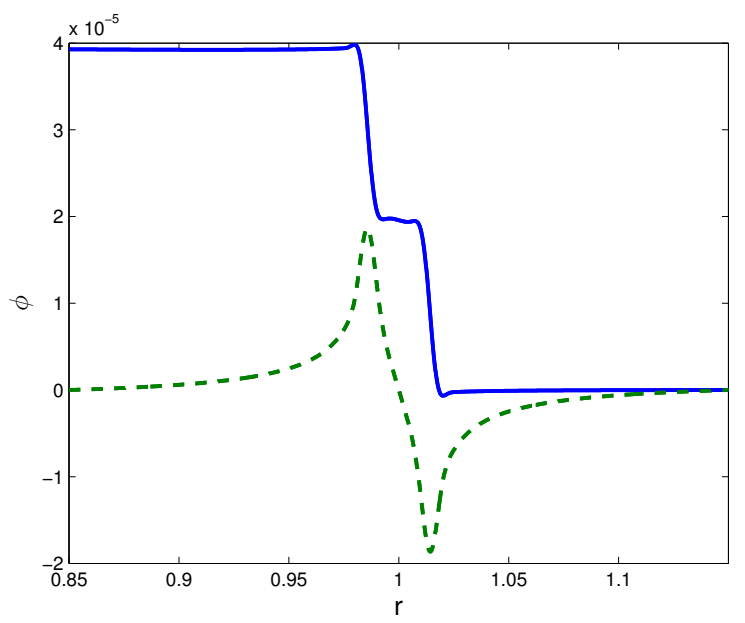

FIG. 3. Profile of the electric potential as a function of $r$, where $r=1$ is the position of the $q=1$ surface, during the linear growth. In solid blue, the real part, in dashed green, the imaginary part.

\section{Coupling}

The coupling between the two regions takes into account two effects. The first one is the influence of the core region on the layer region. It is calculated using Eq. (10) at the boundary between the two regions: the energetic particle term is computed numerically in the core region. In this way a time dependent boundary condition is obtained, fixing the value of the radial derivative of the electric potential at the boundary between the two domains. This value is used as an inner boundary condition to invert the Poisson equation $\Delta_{\perp} \phi=\Omega$ in the annular region. The outer boundary condition is simply given by $\phi=0$.
In the case of a slab geometry, the equation used is not actually Eq. (10), as it is considered that the radius is constant $r=r_{*}$, the equation ends up being

$$
\left.\left[\omega_{A}^{2}(r)-\omega^{2}\right] \partial_{r} \phi\right|_{r_{b}}=-i r_{*} \omega \tilde{\rho}
$$

Noting here that the normalizations are such that $\mu_{0}=$ $\rho_{0}=1$, we can see that the Alfvén frequency is $\omega_{A}(r)=k_{\theta} v_{A, \theta}=k_{\theta} B_{\theta, e q}$. Using that definition for $\omega_{A}$ for the annular region one can express $K$ as $K_{\text {slab }}=$ $\frac{2 \sqrt{2} \pi^{2} L_{S} \mu_{*}^{3 / 2} B_{T}^{5 / 2} e A}{M^{3 / 2} r_{*} \rho_{0} k_{\theta} v_{A, \theta}}$.

The retroaction of the MHD dynamics on the particles' one is thus simply given by the amplitude and phase of $\phi / r$ at the inner boundary. Indeed the MHD evolution provides $\left(\frac{\phi_{e l}}{r\left(P_{\varphi}\right)}\right)_{r\left(P_{\varphi}\right)=r_{b}}$, which is used as the input for the perturbed Hamiltonian. These interactions between the two different domains are done at each time step.

\section{LINEAR NUMERICAL BENCHMARKS}

The numerical results for the linear analysis are presented in Fig. 1-3 In the results presented below $L_{S}$ has been taken to be 2.5. The half-width of the annular layer is $\Delta_{r}=0.15$. That way, the Alfvén frequency continuum varies linearly from $\omega_{A}\left(-\Delta_{r}\right)=-0.06$ to $\omega_{A}\left(\Delta_{r}\right)=0.06$. The distribution function parameters are set as $\beta=20$ and $y_{0}=0.35$ (verifying the narrow gradient condition). For Fig. 1 and Fig. $3, \mu_{*}=2 \times 10^{-2}$, which leads to a theoretical linear frequency $\omega=5.7 \times 10^{-3}$. The small normalized values taken for the dissipation parameters are $\nu=\eta=10^{-9}$.

It can be seen from Fig. 1 that the numerical results fit well the theory in the region linear in $\delta K$. The growth rate is seen to depart from the linear analytic value when $K / K_{0}$ grows far from 1 . This is expected as the growth rate is calculated analytically for $\delta K \ll K_{0}$. It can be seen in Fig. 2 that the numerical mode frequency matches the analytic predictions as well for three cases near threshold, where the resonance frequency is changed via the parameter $\mu_{*}$. If, while keeping $\mu_{*}$ constant, $y_{0}$ is changed, the mode frequency also follows the expected scaling.

It can be seen on the profile shown in Fig. 3 that, as expected, the strong variations of the mode $\phi(r)$ are only present around $r=1(q=1)$. The nearly constant profile on the left of the $q=1$ surface describes, in a slab geometry, a rigid displacement of the plasma core, as expected. Around the $q=1$ surface, one can observe the expected two MHD resonances located at $\omega_{A}(r)=$ $\pm \omega$, whose width depends on the growth rate and on the resistivity and viscosity.

\section{CONCLUSION}

A simplified model for the precessional fishbone instability, based on a separation between the thermal plasma, 
treated with a fluid approach, and an energetic particle population, treated kinetically, has been developed. A linear analytic treatment has been done, allowing us to find a threshold condition, a linear frequency and a linear growth rate for the instability within the scope of our model. It is shown that the threshold value depends on geometric and MHD parameters, as well as energetic particle parameters. A linear numerical study of this model has been performed and is in good agreement with the analytic theory, within the bounds of its validity.

It is shown that the internal kink mode can be destabilized by a negative radial gradient in a deeply trapped energetic particle population. This mode is easier to excite as this gradient gets steeper. The frequency of the mode is determined by the precessional frequency of the particles at the position of steepest gradient. It is seen that the kink mode radial structure, when it has a finite frequency, changes from a single step function centered at the $q=1$ surface to a two-step structure, where the two steps are centered around the Alfvén resonances $\omega_{A}= \pm \omega$.

We calculated an instability threshold condition, showing that it is more easily exceeded with increase in the following:

- the radial density gradient of energetic particles

- the perpendicular velocity

- the radial position of the gradient.

The latter is likely due to the increase of the electric potential with the increase in minor radius. On the other hand, the mode will be stabilized by an increase in magnetic shear that causes a greater continuum damping, and by a higher bulk plasma density.

The longer term objectives of this work are to study the nonlinear evolution of the fishbone mode in a simplified model. The results obtained here have allowed us to benchmark the linear evolution of the mode in the numerical simulations for our model.

\section{Aknowledgements}

The authors would like to thank Dr. Boris Breizman for fruitful discussions. This work has also benefited from discussions within the NLED Enabling Research project (the views and opinions expressed therein do not necessarily reflect those of the european commission). This work was granted access to the HPC resources of AixMarseille Université financed by the project Equip@Meso (ANR-10-EQPX-29-01) of the program "Investissements d'Avenir" supervised by the Agence Nationale pour la Recherche.

${ }^{1}$ L. D. Landau. J. Phys. (USSR) 10, 25, 1946.

${ }^{2}$ T. O'Neil. Phys. Fluids 8, 2255, 1965.

${ }^{3}$ H. L. Berk, B. N. Breizman, and N. V. Petviashvili. Phys. Lett. A 234, 213, 1997.

${ }^{4}$ M. K. Lilley and R. M. Nyqvist. Phys. Rev. Lett. 112, 155002, 2014.

${ }^{5}$ R. G. L. Vann, R. O. Dendy, G. Rowlands, T. D. Arber, and N. d'Ambrumenil. Phys. Plasmas 10, 623, 2003.

${ }^{6}$ H. L. Berk, B. N. Breizman, and H. Ye. Phys. Rev. Lett. 68, 3563, 1992.

${ }^{7}$ F. Zonca, L. Chen, S. Briguglio, G. Fogaccia, G. Vlad, and X. Wang. New J. Phys. 17, 013052, 2015.

${ }^{8}$ K. McGuire, R. Goldston, M. Bell, M. Bitter, K. Bol ad K. Brau, D. Bunhenaur, T. Crowley, S. Davis, and F. Dylla et al. Phys. Rev. Lett. 50, 891, 1983.

${ }^{9}$ L. Chen, R. B. White, and M. N. Rosenbluth. Phys. Rev. Lett. 52, 1122, 1984

${ }^{10}$ A. Ödblom, B. N. Breizman, S. E. Sharapov, and T. C. Henderand V. P. Pastuhkov. Phys. Plasmas 9, 155, 2002.

${ }^{11}$ C. Uberoi. Phys. Fluids 15, $1673,1974$.

${ }^{12}$ B. Coppi and F. Porcelli. Phys. Rev. Lett. 57, 2272, 1986.

${ }^{13} \mathrm{R}$. B. White. The theory of toroidally confined plasmas, Second edition, p.76. Imperial College Press, 2001.

${ }^{14}$ M. Cole, A. Mishchenko, A. Könies, R. Kleiber, and M. Borchardt. Phys. Plamsas 21, 072123, 2014.

${ }^{15}$ G. Y. Fu, W. Park, H. R. Strauss, J. Breslau, J. Chen, S. Jardin, and L. E. Sugiyama. Phys. Plasmas 13, 052517, 2006.

${ }^{16}$ F. Zonca, P. Buratti, A. Cardinali, L. Chen, J.-Q. Dong, Y.-L. Long, A. V. Milovanov, F. Romanelli, P. Smeulders, and L. Wang et al. Nucl. Fusion 47, 1588, 2007.

${ }^{17}$ G. Vlad, S. Briguglio, G. Fogaccia, F. Zonca, V. Fusco, and X. Wang. Nucl. Fusion 53, 083008, 2013.

${ }^{18} \mathrm{M}$. Abramowitz and I. Stegun. Handbook of mathematical functions, p.297. Dover publications, 1972.

${ }^{19}$ T. Nakamura, R. Tanaka, T. Yabe, and K. Takizawa. J. Comput. Phys. 174, 171, 2001.

${ }^{20}$ M. Lesur, Y. Idomura, and X. Garbet. Phys. Plasmas 16, 092305, 2009.

${ }^{21}$ O. Agullo, M. Muraglia, A. Poyé, S. Benkadda, M. Yagi, X. Garbet, and A. Sen. Phys. Plasmas 21, 092303, 2014. 